\title{
Which treatment for low back pain? A factorial randomised controlled trial comparing intravenous analgesics with oral analgesics in the emergency department and a centrally acting muscle relaxant with placebo over three days [ISRCTN097 I 9705] Christof Havel ${ }^{1}$, Anna Sieder ${ }^{1}$, Harald Herkner ${ }^{1}$, Hans Domanovits ${ }^{1}$, Mascha Schmied ${ }^{2}$, Rudolf Segel3, Maria Koreny ${ }^{1}$, Anton N Laggner ${ }^{1}$ and Marcus Müllner*1
}

\author{
Address: ${ }^{1}$ Univ. Klinik fur Notfallmedizin, Allgemeines Krankenhaus Wien, Währinger Gürtel 18-20, A-1090 Wien, Austria, ${ }^{2}$ Univ.-Klinik fur \\ Neurologie, Allgemeines Krankenhaus Wien, Währinger Gürtel 18-20, A-109o Wien, Austria and 3Hospital Pharmacy, Allgemeines \\ Krankenhaus Wien, Währinger Gürtel 18-20, A-1090 Wien, Austria \\ E-mail: Christof Havel - Christof.Havel@univie.ac.at; Anna Sieder - Anna.Sieder@univie.ac.at; Harald Herkner - Harald.Herkner@akh- \\ wien.ac.at; Hans Domanovits - Hans.Domanovits@akh-wien.ac.at; Mascha Schmied - Mascha.Schmied@univie.ac.at; \\ Rudolf Segel - Rudolf.Segel@akh-wien.ac.at; Maria Koreny - Maria_Koreny@hotmail.com; Anton N Laggner - Anton.Laggner@akh- \\ wien.ac.at; Marcus Müllner* - Marcus.Muellner@univie.ac.at \\ *Corresponding author
}

This article is available from: http://www.biomedcentral.com/I47I-227X/I/2

(c) 200I Havel et al; licensee BioMed Central Ltd. Verbatim copying and redistribution of this article are permitted in any medium for any non-commercial purpose, provided this notice is preserved along with the article's original URL. For commercial use, contact info@biomedcentral.com

\begin{abstract}
Background: About two thirds of adults suffer from backpain at some time during their life. In the emergency room many patients with acute back pain are treated with intravenous nonsteroidal analgesics. Whether this treatment is superior to oral administration of non-steroidal analgesics is unknown. Intravenous administration, however, requires considerable amounts of resources and accounts for high workload in busy clinics. In the further course centrally acting muscle relaxants are prescribed but the effectiveness remains unclear. The objective of this study is on the one hand to compare the effectiveness of intravenous with oral non-steroidal analgesics for acute treatment and on the other hand to compare the effectiveness of a centrally active muscle relaxant with placebo given for three days after presentation to the ED (emergency department).

Methods/Design: This study is intended as a randomised controlled factorial trial mainly for two reasons: $(I)$ the sequence of treatments resembles the actual proceedings in every-day clinical practice, which is important for the generalisability of the results and (2) this design allows to take interactions between the two sequential treatment strategies into account. There is a patient preference arm included because patients preference is an important issue providing valuable information: ( $I$ ) it allows to assess the interaction between desired treatment and outcome, (2) results can be extrapolated to a wider group while (3) conserving the advantages of a fully randomised controlled trial.
\end{abstract}

Conclusion: We hope to shed more light on the effectiveness of treatment modalities available for acute low back pain. 


\section{Background}

The estimated prevalence of back pain in the Viennese population is about $12 \%$ [1]. Accordingly, a recent comprehensive review article reports that about two thirds of adults suffer from low back pain and back pain at some time. This makes back pain one of the most frequent symptoms why physicians are consulted, surpassed only by respiratory symptoms [2].

About 30 patients present to our emergency department every week because of acute low back pain and many of these are treated with intravenous non-steroidal analgesics. Whether this treatment is superior to oral administration non-steroidal analgesics is unknown. Intravenous administration, however, requires considerable amounts of resources and accounts for high workload in busy clinics.

In the further course non-steriodal analgesics are usually prescribed, often together with centrally active muscle relaxants. There is evidence of a possible beneficial effect, but the quality of the existing studies is poor [3]. Accordingly, some specialists say that the effectiveness of centrally active muscle relaxants is unclear [2].

\section{Objectives of the study}

1) To compare the effectiveness of intravenous non-steroidal analgesics with oral non-steroidal analgesics for acute treatment in the emergency department (Stage 1)

2) To compare the effectiveness of either arm with the effectiveness in those patients who refused to be randomised but agreed to participate in the patient preference trial with the treatment of their choice

3) To compare the effectiveness of a centrally active muscle relaxant with placebo given for three days after presentation to the ED (Stage 2)

4) To compare the effectiveness of either arm with the effectiveness in those patients who refused to be randomised but agreed to participate in the patient preference trial with the treatment of their choice

\section{Methods/Design}

This study is intended as a randomised controlled factorial trial mainly for two reasons: (1) the sequence of treatments resembles the actual proceedings in everyday clinical practice, which is important for the generalisability of the resultsand (2) this design allows to take interactions between the two sequential treatment strategies into account. The patient preference arm is included because patients preference is an important issue providing valuable information: (1) it allows to assess the interaction between desired treatment and outcome, (2) results can be extrapolated to a wider group while (3) conserving the advantages of a fully randomised controlled trial [4].

\section{Study population}

Patients with acute low back pain attending the emergency department of the Vienna General Hospital, a 200o-bed teaching hospital. The emergency department has a busy outpatient unit with ten rooms for use by general internists, neurologists, surgeons, orthopaedics, dermatologists, jaw surgeons, gynaecologists, ophthalmologists and ear-nose-throat physicians [5]. Each year, about 70 ooo patients attend the emergency department, of which 30 are treated for acute low back pain every week.

It is well known that a precise anatomical diagnosis is difficult and often not possible. Therefore we aim for a pragmatic approach which is intended (1) to rule out systemic disease, such as neoplasm, infection and inflammatory arthritis, accounting for about $3 \%$ of the cases [1] and (2) to detect neurological compromise that may require neuro-surgical evaluation/intervention. It is generally advised not to perform any form of imaging if non of our exclusion criteria (see below) is present [6].

\section{Inclusion criteria}

- lower back pain localised between $12^{\text {th }}$ rib and gluteal fold

- duration of pain of the current period $<7$ days

- attending the Department of Emergency Medicine at the Vienna General Hospital because of low back pain

- agree to be randomised or agree to be included in the patient preference trial

- written informed consent

\section{Exclusion criteria}

- History:

- ingestion of any analgesic drug within 6 hours

- direct impact trauma

- history of cancer

- unexplained weight loss (> $10 \mathrm{~kg}$ within 3 months)

- current injection drug use

- any known chronic infection, such as Hepatitis, HIV (Human Immuno-deficiency Virus), tuberculosis 
- immunosuppressive therapy (such as systemic corticosteroids, ciclosporine, or such)

- organ transplantation

- history of inflammatory arthritis of large joints

- current bowel or bladder dysfunction

- involved in litigation

- alcohol abuse

- age $<19$ and $>65$ years

- current abdominal problems (epigastric pain)

- a history of gastric or duodenal ulcer

- a history of severe renal or hepatic insufficiency or severe coronary insufficiency.

- Allergies against any NSA (non-steroidal analgesics) or tizanidin

- Physical examination:

- Fever $>38^{\circ} \mathrm{C}$

- sensory or motor deficit in lower limb

- Lasegue positive $>60^{\circ}[7]$

- Pregnancy: to be ruled out by a commercially available and routinely used urine pregnancy test (DIPRO hCGCARD $^{\circledR}$, Dipro Diagnostic Products, Ebreichsdorf, Austria)

- Urinary tact infection: to be ruled out by a commercially available and routinely used urine dip stick test (COMBUR 9-Test ${ }^{\circledR}$, Roche, Mannheim, Germany); infection is assumed if nitrite and leukocytes test positive

\section{- Communication problems:}

- Patients who appear not to be able to understand the information provided to give informed consent for participation in this trial because of mental or physical handicaps. It is the duty of the enrolling physician to decide whether a potential participant has sufficient language skills to understand the information provided or to communicate that she/he understands.

- Patients who appear not to be able to understand the information provided to give informed consent for partici- pation in this trial because of language barriers. Those who need an interpreter to communicate with the treating physician are certainly ineligible. Otherwise it is the duty of the enrolling physician to decide whether a potential participant has sufficient language skills to understand the information provided.

\section{Ethical approval}

The study will be performed in accordance with the Declaration of Helsinki (1964), including current revisions, the Austrian „Arzneimittelgesetz" (1994) and the GCP guidelines of the European Commission. The protocol was approved by the ethics committee of the Vienna Medical Faculty. Depending on the form of consent patients will be enrolled in the randomised trial or the patient preference arm.

\section{Planned intervention and timing (figure I)}

After consent and enrolment patients are randomised to the stage one intervention.

\section{Stage I intervention}

Patients are randomised to oral diclofenac (150 mg) plus intravenous placebo or oral placebo plus intravenous diclofenac $(75 \mathrm{mg})$ in a double blind fashion. The dose of diclofenac for oral administration has to be twice the intravenous dose because of a $50 \%$ first pass elimination of the active ingredient. Patients will be assessed immediately before and $90 \mathrm{~min}$ after treatment (this is the average time needed for the intravenous administration of an infusion containing analgesics). The primary endpoint at 90 minutes is pain assessed with a visual analogue scale (VAS) [8], secondary endpoint is the Roland Morris disability questionnaire (RMDQ) [9], and the quality of life score as measured by the short form 36 (SF-36) [10]. If patients still have severe pain further intravenous therapy should be given according to the discretion of the treating physician. Patients who refuse randomisation but agree to participate in the patient preference trial will receive the treatment of their choice. Treatment and follow-up will be identical in the randomised and patient preference groups.

\section{Stage 2 intervention}

After the first step patients are randomised to oral diclofenac $(2 \times 100 \mathrm{mg}$ per day or $1 \times 100 \mathrm{mg}$ per day if bodyweight is $<60 \mathrm{~kg}$ ) and the oral muscle-relaxant tizanidin (Sirdalud ${ }^{\circledR} 3 \times 2 \mathrm{mg}$, Novartis Pharma AG, Basel, Switzerland) over 3 days versus oral diclofenac $(2 \times 100 \mathrm{mg}$ per day or $1 \times 100 \mathrm{mg}$ per day if bodyweight is $<60 \mathrm{~kg}$ ) plus oral placebo in a double blind fashion. Patients with a history of gastric pain, suggestive of ulcer or non-ulcer dyspeptic disease will receive a proton pump inhibitor for gastric protection (pantoprazol $1 \times 40 \mathrm{mg}$ per day, Pantoloc ${ }^{\circledR}$ Byk Pharma Österreich). 


\section{Acute low backpain}

\section{Randomisation}
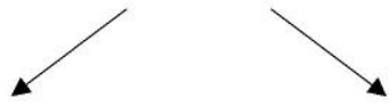

Immediately

Diclofenac $150 \mathrm{mg}$ po

$\mathrm{n}=115$
Immediately

Diclofenac $75 \mathrm{mg}$ iv

$\mathrm{n}=115$

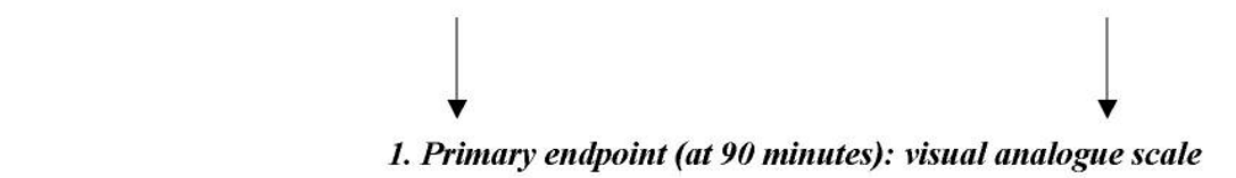

Randomisation

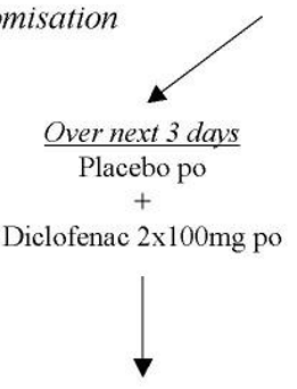

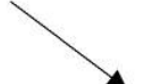

Over next 3 davs

Tizanidin $3 \times 2 \mathrm{mg}$ po

Diclofenac $2 \times 100 \mathrm{mg}$ po

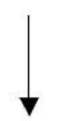

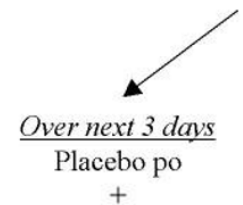

Diclofenac 2x100mg po

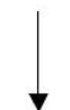

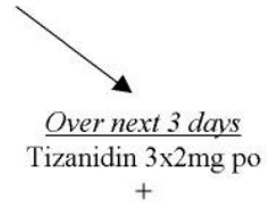

Diclofenac 2x100mg po

2. Primary endpoint (at day 4): visual analogue scale

Figure I

Patient flow. Planned interventions and timing of interventions; po indicates enteral (peroral) administration, iv indicates intravenous administration

As in the first step, patients who refuse randomisation but agree to participate in the patient preference trial will receive the treatment of their choice. Treatment and follow-up will be identical in the randomised and patient preference groups.

\section{Sample size}

Based on a clinically relevant difference of $10 \mathrm{~mm} \pm 20$ $\mathrm{mm}$ in the visual analogue scale the projected sample size at a two-sided p-value of 0.02 and a power of 0.85 is 92 per group. This effect size is an estimate based on the outcome of pain measurement 60 minutes after the oral administration of a non-steroidal analgesic and a COX-2 inhibitor in patients with chronic low back pain. [11] We do not assume a relevant attrition rate for phase 1.
Taking a 20\% attrition rate over the next 4 days into account the projected sample size is 115 per group to reliably detect such a difference at the end of phase 2 .

Sample size estimations are based on sample size tables for clinical studies [12].

\section{Statistical analysis}

We intend to use two models: (1) a model containing only randomised patients and (2) a model containing randomised patients and patients in the patient preference arms. Data will be analysed in an intention-to-treat fashion if patients receive a treatment other than the allocated treatment For group comparison of continuous data in model 1 (randomised patients only) either a two tailed t-test, and an analysis of covariance to adjust for baseline values in the VAS, or, in case of a non-normal distribu- 
tion, or ordinal data (RMDQ, SF-36), the Mann-Whitney U-Test will be used. For model 2 (randomised patients and patients with preferred treatment) multiple linear regression will be used to account for baseline differences and the randomisation status [4]. Stata version 7 (Stata Corporation, TX, USA) will be used for data analysis. A p-value $<0.05$ is assumed to indicate evidence against the null hypothesis.

We believe that it is not necessary to define stopping rules as the observational period is very short and we do not expect that any treatment, which is already widely used, will result in increased mortality or disability.

\section{Assignment}

Unit of randomisation and method to generate allocation (for both stages)

The unit of randomisation is the individual patient. Blockwise randomisation in blocks of 4 is intended to achieve intervention and control groups of equal size [13]. Allocation will be performed using computer programme (Machin D, Campbell MJ. Sampsize V2.0 1997) [12].

\section{Timing of allocation and concealment of allocation}

Immediately after consent to participate and enrolment a consecutively numbered, opaque and sealed envelope containing an allocation number (see below) will be opened. Treatment (oral $\mathrm{v}$ intravenous diclofenac) will commence immediately afterwards. For the second stage of the trial patients will be randomised to a centrally active muscle relaxant or placebo. Both groups should receive a long acting non-steroidal analgesic (diclofenac 2 $\times 100 \mathrm{mg} \mathrm{po}$ ) and will be evaluated after 4 days. Randomisation and allocation again will be performed as described above with opaque, sealed envelopes.

\section{Method to separate generator from executor}

The randomisation will be prepared by a person not involved in recruitment, treatment or assessment of patients

\section{Masking}

Stage 1

This trial is intended to be double blind. Patients, doctors and outcome assessors are blinded to the nature of the intervention. Each patient is to receive a pre-prepared intravenous administration of $250 \mathrm{ml} \mathrm{NaCI} 0.9 \%$ containing either $75 \mathrm{mg}$ diclofenac or placebo of equal appearance. At the same time patients will receive a capsule containing $150 \mathrm{mg}$ diclofenac or placebo. The placebo capsule contains lactose powder and has the same size, colour and taste as the active drug.

\section{Stage 2}

The second stage is also intended as a double blind trial. Both groups will receive oral diclofenac (Dolpasse ${ }^{\circledR}$, Fresenius, $2 \times 100 \mathrm{mg}$ ) for 3 days. Patients are randomised either to placebo $3 \times 1$ for 3 days or oral tizanidin (Sirda$\operatorname{lud}^{\circledR} 3 \times 2 \mathrm{mg}$ ) $3 \times 1$ for 3 days. The placebo capsule contains lactose powder and has the same size, colour and taste as the active drug.

\section{Was blinding successful?}

To assess whether blinding was successful we will ask patients and outcome assessors whether the patient received active enteral or parenteral treatment (Stage 1). The question intended for the patient, and the outcome assessor alike, is: "Do you think the active treatment was the infusion or the capsule?" Possible answers are: "Most likely in the infusion."; "Don't know."; "Most likely in the capsule." For stage 2 we will ask the patient and the outcome assessor: "Do you think you received the active treatment or the inactive treatment?" Possible answers are: "Most likely yes."; "Don't know."; "Most likely no."

\section{Baseline measurements}

We intend to assess following baseline parameters besides the clinical symptoms leading to inclusion in the study and the outcome parameters: age, gender, highest education.

\section{Primary and secondary outcome measures}

For both stages the primary outcome measure is the pain assessed by means of the VAS [8]. Patients receive an A4 sheet with a $10 \mathrm{~cm}$ bold line in the centre which is has "no pain" above the left end of the line and "pain as bad as you have ever experienced" at the right end of the line. Patients are advised to set a marker at the applicable range with a pencil. The distance of the marker from "no pain" $(=0)$ will be measured and expressed in $\mathrm{mm}$ (maximum $=100$ ). For stage one, the visual analogue scale is evaluated immediately before and $90 \mathrm{~min}$ after treatment, for stage two, the VAS is evaluated on day 4 (=three days after enrolment). Patients will be advised to fill out the scale at home and return the original sheet by a prepaid return postage envelope.

For both stages the RMDQ [9] and the SF-36 [10] are employed in an adapted form for secondary outcome measurements. The Roland Morris disability questionnaire [14] is used in the cross cultural adapted and validated form for German-speaking patients [10]. The questionnaire is a questionnaire with 24 items that reflect varied activities of daily living due to back pain. An item receives a score of 1 if it is checked as applicable by the patient and a score of $\mathrm{o}$ if not marked. Accordingly scores can vary from o (no disability) to 24 (severe disability). 
The SF-36 is used in the cross cultural adapted and validated form for German-speaking patients [10]. We selected the items 1 (general health perception), 2 (change in health), 4 (role limitations (physical problems)), 5 (role limitations (emotional problems)), 9 (vitality) and 10 (social functioning). The score will be calculated according to the test-manual

The RMDQ and the SF 36 is evaluated immediately before treatment (stage 1) by personal interview, then, for stage two, the RMDQ and the SF 36 is evaluated 4 days after enrolment. Patients will be interviewed by telephone using the structured format of the questionnaires of RMDQ and SF-36.

For patients with persisting pain we will recommend (1) to continue analgesic therapy and (2) to attend their general practitioner for further diagnostic work-up and treatment.

\section{Adverse events}

An adverse event is any event during a clinical study, including intercurrent illness or accident, which impairs the well-being of the subject; it may also take the form of an abnormal laboratory value. The term adverse event does not imply a causal relationship with the study treatment.

All subjects experiencing adverse events - whether considered associated with the use of the study medication or not - will be monitored until symptoms subside and any abnormal laboratory values have returned to baseline, or until there is a satisfactory explanation for the changes observed, or until death, in which case a full pathologist's report will be supplied, if possible. All findings must be reported on an "Adverse event" page in the case record form.

Adverse events are divided into the categories "serious" (fatal, life-threatening, or permanently disbling) and "nonserious". All adverse events, including intercurrent illnesses, will be reported and documented.

\section{Data management}

We intend to transfer data from case record forms to the computer database (MS Excel 97) by two investigators in duplicate and independently. Data will be compared and inconsistencies will be sorted out by referring to the original case record form. If there are still range, consistency or plausibility problems, the patient chart will be assessed. If after these measures values are still implausible or missing we intend to treat them as missing data. We do not intend to substitute these data with e.g. average values. We plan to assess whether missing data, par- ticularly core data, may introduce bias by sensitivity analysis.

\section{Confidentiality}

Upon entry into the trial each patient will receive a unique identifying number. Subsequently only this number will be used in the case record forms and the investigator responsible for data management is not aware of the patients' identity nor treatment allocation. The patient's identity is only known to the treating physician and those investigators who are responsible for followup management. These investigators are however not in the possession of any other confidential information.

\section{Abbreviations \\ RMDQ Roland Morris disability questionnaire}

SF-36 Short Form 36 health survey questionnaire

VAS Visual Analogue Scale

ED Emergency Department

HIV Human Immuno-deficiency Virus

NSA Non-Steroidal Analgesics

\section{Competings interests}

None of the investigators will earn in any possible way by the conduct of this trial and more importantly, analysis, interpretation and publication of the data will be performed independently by the investigators and will not be influenced by the pharmaceutical industry.

There are no other competing interests.

\section{Acknowledgements}

We submitted the protocol to the "Medizinisch-Wissenschaftlicher Fonds des Bürgermeisters der Bundeshauptstadt Wien". Up to now (17.10.0I) there is no decision.

NOVARTIS Pharma GmbH will support the study by providing the patient insurance.

NOVARTIS Pharma GmbH will not be involved in data collection, analysis or data interpretation.

We are grateful to Dr.Martin Schillinger for generating the random sequence.

\section{References}

I. Statistisches Zentralamt: Gesundheitsbericht der Stadt Wien 2000 [Health report of Vienna 2000]. Vienna 2000

2. Deyo RA, Weinstein JN: Low back pain. N Engl J Med 200I, 344:363-370

3. van Tulder MW, Scholten RJPM, Koes BW, Deyo RA: Non steroidal anti-inflammatory drugs for low back pain (Cochrane Rev) The Cochrane Library 2000, issue 4.:

4. Torgerson D, Sibbald B: Understanding controlled trials: What is a patient preference trial? $B M J 1998,316: 360$ 
5. Bur A, Müllner M, Sterz F, Hirschl MM, Laggner AN: The emergency department in a 2000-bed teaching hospital: saving open ward and intensive care facilities. European Journal of Emergency Medicine 1997, 4:19-33

6. Kendrick D, Fielding K, Bentley E, Kerslake R, Miller P, Pringle M: Radiography of the lumbar spine in primary care patients with low back pain: a randomised controlled trial. BMJ 200I, 322:400-405

7. Hellmann DB, Stone $\mathrm{JH}$ : Arthritis and musculoskeletal disorders. In: Current medical diagnosis and treatment. Tierney LM, McPhee SJ, Papadakis MA (eds). McGraw Hill New York 40th edition 2001825

8. Carlsson AM: Assessment of chronic pain I. Aspects of the reliability and validity of the visual analogue scale. Pain 1983, | 76:87-10|

9. Wiesinger GF, Nuhr M, Quittan M, Ebenbichler G, Wölfl G, FialkaMoser V: Cross-cultural adaptation of the Roland-Morris questionnaire for German-speaking patients with low back pain. Spine 1999, 24:1099-1103

10. Bullinger M, Kirchberger I, Ware J: Der deutsche SF-36 Health Survey. Zf Gesundheitswiss 1995, 3:21-36

II. Babej-Dölle R, Freytag S, Eckmeyer J, Zerle G, Schinzel S, Schmieder G, Stankov G: Parenteral dipyrone versus diclophenac and placebo in patients with acute lumbago or sciatic pain: Randomized observer-blind mnlticenter study. Int J Clin Pharmacology 1994, 32:204-209

12. Machin D, Campbell MJ: Sample size tables for clinical trials. Blackwell Science, UK 1997

13. Altman DG, Bland M]: How to randomise. BM] 1999, 3 | 9:703-704

14. Roland M, Morris R: A study of the natural history of low back pain. Part I: development of a reliable and sensitive measure of disability in low back pain. Spine 1983, 8:|4|-| 44

Publish with BioMed Central and every scientist can read your work free of charge

"BioMedcentral will be the most significant development for disseminating the results of biomedical research in our lifetime."

Paul Nurse, Director-General, Imperial Cancer Research Fund

Publish with BMC and your research papers will be:

- available free of charge to the entire biomedical community

- peer reviewed and published immediately upon acceptance

- cited in PubMed and archived on PubMed Central

- yours - you keep the copyright
BioMedcentral.com editorial@biomedcentral.com 